\title{
On the Principle of Limiting Absorption for the Dirac Operator
}

By

Osanobu YAMADA*

\section{§1. Introduction}

In this paper we are concerned with the Dirac operator

$$
L=\frac{1}{i} \sum_{j=1}^{3} \alpha_{j} \frac{\partial}{\partial x_{j}}+\beta+Q(x)
$$

with a Hermitian symmetric potential $Q(x)$ in $\mathbb{R}^{3}$, which appears in the relativistic quantum mechanics. The $\alpha_{j}$ and $\beta$ are the so-called Dirac matrices. The mathematical scattering problem for the Dirac operator has been investigated by many authors (see, e.g., Birman [2], Kato [6], Mochizuki [7], Prosser [8], Roze [9]). Our aim in the present paper is to study the nature of the spectrum of the Dirac operator by means of the principle of limiting absorption. Roughly speaking, this principle may be defined to research a certain limit of $u_{\lambda+i \mu}$ as $\mu \downarrow 0$ or $\mu \uparrow 0$, where $u_{\lambda+i \mu}$ is a solution of the equation

$$
(L-(\lambda+i \mu)) u=f
$$

(see Eidus [4]). Eidus [3], [4] developed a principle of limiting absorption for elliptic Dirichlet problems in exterior domains, where the radiation conditions at infinity and the near-singularity properties of fundamental solutions were made use of in an essential manner. But recently Agmon [1] proposed a new method without explicit recourse to these tools and involving a priori estimates, which is valid for elliptic operators in the

Received August 16, 1972.

Communicated by S. Matsuura.

* Department of Mathematics, Kyoto University, Kyoto, Japan. 
whole space under appropriate decay conditions on the coefficients at infinity. We shall derive results analogous to Agmon's for the Dirac operator provided that the potential $Q(x)$ decreases near infinity like $|x|^{-1-h}, h>0$.

We shall outline the contents of the present paper. In $\S 2$ we give some fundamental spectral properties of the Dirac operator, such as essential self-adjointness, invariance of essential spectra and non-existence of eigenvalues $\lambda$ with $|\lambda|>1$. In $\S 3$ we shall estimate the resolvent of the unperturbed Dirac operator in terms of Fourier transforms, which is essentially due to Agmon [1]. In $\S 4$ we show that the principle of limiting absorption holds for the Dirac operator satisfying the above condition and prove the absolute continuity.

\section{§ 2. The Dirac Operator}

We consider the unperturbed Dirac operator

$$
L_{0}=\frac{1}{i} \sum_{j=1}^{3} \alpha_{j} \frac{\partial}{\partial x_{j}}+\beta, \quad i=\sqrt{-1}
$$

for $x=\left(x_{1}, x_{2}, x_{3}\right) \in \mathbb{R}^{3}$, where $\alpha_{j}, \beta$ are $4 \times 4$ constant Hermitian matrices and satisfy the anti-commutation relations

$$
\alpha_{j} \alpha_{k}+\alpha_{k} \alpha_{j}=2 \delta_{j k} I, \quad j, k=1,2,3,4
$$

with the convention $\alpha_{4}=\beta$ ( $I$ is the unit $4 \times 4$ matrix and $\delta_{j k}$ is Kronecker's delta). Since $L_{0}$ is a formal differential operator, we can construct from $L_{0}$ various operators in the Hilbert space $L^{2}=\left(L^{2}\left(\mathbf{R}^{3}\right)\right)^{4}$ consisting of all $\mathbf{C}^{4}$-valued functions such that

$$
\|u\|_{L^{2}}^{2}=\int_{\mathbf{R}^{3}}|u(x)|^{2} d x<+\infty,|u(x)|^{2}=\sum_{j=1}^{4}\left|u_{j}(x)\right|^{2}
$$

The associated inner product is

$$
(u, v)=\int_{\mathbf{R}^{3}}\left\langle u(x), v(x)>d x,\left\langle u(x), v(x)>=\sum_{j=1}^{4} u_{j}(x) \overline{v_{j}(x)} .\right.\right.
$$

We denote by $T_{0}$ the symmetric operator $T_{0} u=L_{0} u$ with the domain 
$D\left(T_{0}\right)=C_{0}^{\infty} . \quad C_{0}^{\infty}$ consists of all $\mathbf{C}^{4}$-valued functions $u(x)$ with the components $u_{j}(x)$ lying in $C_{0}^{\infty}\left(\mathbf{R}^{3}\right)$, all $C^{\infty}$ functions on $\mathbf{R}^{3}$ with compact support. Then by Kato [6], p. 306, $T_{0}$ turns out essentially self-adjoint and the domain of its self-adjoint realization $H_{0}$ is $H^{1} . H^{1}$ denotes the space of all $L^{2}$ functions with square integrable first order distribution derivatives.

The following proposition is well-known (see, e.g., Mochizuki [7]).

Proposition 2.1. Every real $\lambda$ such that $|\lambda| \geqq 1$ is in the continuous spectrum of $H_{0}$ and the interval $(-1,+1)$ is contained in the resolvent set of $H_{0}$.

Now we consider the Hermitian matrix

$$
\widehat{L}_{0}(\xi)=\sum_{j=1}^{3} \alpha_{j} \xi_{j}+\beta
$$

for $\xi=\left(\xi_{1}, \xi_{2}, \xi_{3}\right) \in \mathbb{R}^{3}$. The following property of $\hat{L}_{0}(\xi)$ can be proved by using the relation (2.1) and will be applied in $\S 4$.

Proposition 2.2. The following relation holds for $\hat{L}_{0}(\xi)$ :

$$
\left(\widehat{L}_{0}(\xi)\right)^{2}=\left(|\xi|^{2}+1\right) I \text {. }
$$

The eigenvalues of $\hat{L}_{0}(\xi)$ are $+\sqrt{|\xi|^{2}+1}$ and $-\sqrt{|\xi|^{2}+1}$ with multiplicity 2.

Next let us consider the perturbed Dirac operator

$$
L=L_{0}+Q(x)=\frac{1}{i} \sum_{j=1}^{3} \alpha_{j} \frac{\partial}{\partial x_{j}}+\beta+Q(x)
$$

for $x=\left(x_{1}, x_{2}, x_{3}\right) \in \mathbf{R}^{3}$, where $Q(x)$ is a $4 \times 4$ Hermitian matrix valued function. For the self-adjointness of the perturbed Dirac operator, Prosser [8] proved the following.

Proposition 2.3. Suppose that $|Q(x)|$ lies in $L^{p}\left(\mathbf{R}^{3}\right)(p>3)$, where $|Q(x)|^{2}=\sum_{i, j=1}^{4}\left|q_{i j}(x)\right|^{2}$ for $Q(x)=\left(q_{i j}(x)\right)$ and $L^{p}\left(\mathbb{R}^{3}\right)$ denotes all complexvalued measurable functions $f(x)$ defined in $\mathbb{R}^{3}$ such that $\left(\|f\|_{\left.L^{p}\right)^{p}}=\int_{\mathbf{R}^{3}}\right.$ 
$|f(x)|^{p} d x<+\infty$. Then for any given $\varepsilon>0$, there exists a positive constant $C_{\varepsilon}$ such that

$$
\|Q u\|_{L^{2}} \leqq\||Q|\|_{L^{p}}\left(\varepsilon\left\|H_{0} u\right\|_{L^{2}}+C_{\varepsilon}\|u\|_{L^{2}}\right),
$$

for all $u \in D\left(H_{0}\right) . \quad H=H_{0}+Q$ is self-adjoint with the domain $D(H)=$ $D\left(H_{0}\right)=H^{1}$.

We denote by $L^{p}(\Omega)$ the class of all $\mathbb{C}^{4}$-valued functions $f(x)$ such that $\left(\|f\|_{L^{p}(\Omega)}\right)^{p}=\int_{\Omega}|f(x)|^{p} d x<+\infty$, where $\Omega$ is an open set in $\mathbb{R}^{3}$ and denote by $H^{1}(\Omega)$ the class of all $L^{2}(\Omega)$ functions with first order distribution derivatives in $L^{2}(\Omega)$.

Corollary 2.1. Let $p>3$. For any given $\varepsilon>0$, there exists a positive constant $C_{\varepsilon}^{\prime}$ not depending on $R>0$ such that

$$
\|Q u\|_{L^{2}\left(B_{R}\right)} \leqq\||Q|\|_{L^{p}\left(B_{R+1}\right)}\left(\varepsilon\left\|L_{0} u\right\|_{L^{2}\left(B_{R+1}\right)}+C_{\varepsilon}^{\prime} \| u_{L^{2}\left(B_{R+1}\right)}^{\prime}\right)
$$

for all $u \in H^{1}\left(B_{R+1}\right)$, where $B_{R}$ denotes the ball with the center at the origin and the radius $R$.

Proof. We take $\beta_{R}(x) \in C_{0}^{\infty}\left(\mathbb{R}^{3}\right)$ such that

$$
\begin{gathered}
\beta_{R}(x)=\left\{\begin{array}{l}
1,|x| \leqq R \\
0,|x| \geqq R+\frac{1}{2},
\end{array}\right. \\
\max _{x \in \mathbf{R}^{3}}\left|\beta_{R}(x)\right|+\sum_{j=1}^{3}\left|\frac{\partial}{\partial x_{j}} \beta_{R}(x)\right| \leqq C_{1},
\end{gathered}
$$

where $C_{1}$ is a constant not depending on $R$. Then we have by Proposition 2.3

$$
\|Q u\|_{L^{2}\left(B_{R}\right)} \leqq\left\|\beta_{R} Q \cdot \beta_{R} u\right\|_{L^{2}} \leqq\left\|\mid \beta_{R} Q\right\| \|_{L^{p}}\left(\varepsilon\left\|_{L_{0}}\left(\beta_{R} u\right)\right\|_{L^{2}}+C_{\varepsilon}\left\|\beta_{R} u\right\|_{L^{2}}\right),
$$

for all $u \in H^{1}\left(B_{R+1}\right)$. Thus the proof of (2.4) is complete.

Q.E.D.

The following proposition states a relation between the essential spectra of $H_{0}$ and $H=H_{0}+Q$. 
Proposition 2.4. (cf. Birman [2]). Assume that $|Q(x)|$ is a locally $L^{p}$ function $(p>3)$ and $|Q(x)|=o(1)(|x| \rightarrow \infty)$. Then the essential spectra of $H$ and $H_{0}$ coincide, i.e., every real number $\lambda$ such that $|\lambda| \geqq 1$ is in the essential spectrum of $H$, and any spectrum of $H$ in $(-1,+1)$, if it exists, is discrete.

Proof. According to Kato [6], Theorem IV-5.35, it suffices to prove that

$$
\left(H_{0}-i\right)^{-1}-(H-i)^{-1}=(H-i)^{-1} Q\left(H_{0}-i\right)^{-1}
$$

is completely continuous. Let $\left\{\varphi_{k}\right\}$ be an arbitrary infinite sequence such that $\left\|\varphi_{k}\right\|_{L^{2}} \leqq$ const., $k=1,2, \ldots$. We put $\psi_{k}=\left(H_{0}-i\right)^{-1} \varphi_{k}$. Then $\psi_{k} \in$ $D\left(H_{0}\right)=H^{1}$, and $\left\{H_{0} \psi_{k}\right\},\left\{\psi_{k}\right\}$ are bounded sequences in $L^{2}$, which implies that $\left\{\psi_{k}\right\}$ is a bounded sequence in $H^{1}$. By Rellich's theorem we can select a subsequence $\left\{\psi_{k}^{\prime}\right\}$ from the sequence $\left\{\psi_{k}\right\}$ which converges locally in $L^{2}$ to some function. Then we obtain by Corollary 2.1

$$
\begin{aligned}
& \left\|Q \psi_{j}^{\prime}-Q \psi_{k}^{\prime}\right\|_{L^{2}} \leqq\left\|Q \psi_{j}^{\prime}-Q \psi_{k}^{\prime}\right\|_{L^{2}\left(B_{R}\right)}+\left\|Q \psi_{j}^{\prime}-Q \psi_{k}^{\prime}\right\|_{L^{2}\left(E_{R}\right)} \\
& \leqq \\
& \quad+\left(\sup _{x \in E_{R}}|Q(x)|\right)\|\|_{L^{p}\left(B_{R+1}\right)}\left(\varepsilon\left\|L_{0} \psi_{j}^{\prime}-L_{0} \psi_{j}^{\prime}\right\|_{L^{2}\left(B_{R+1}\right)}+\psi_{\varepsilon}^{\prime}\left\|\psi_{j}^{\prime}\right\|_{L^{2}\left(E_{R}\right)},\right.
\end{aligned}
$$

where $E_{R}$ denotes the complement of $B_{R}$. Thus it follows from the assumption on $Q(x)$ that $\left\{Q \psi_{k}^{\prime}\right\}$ is a Cauchy sequence in $L^{2}$. As $(H-i)^{-1}$ is a bounded operator on $L^{2},\left\{(H-i)^{-1} Q \psi_{k}^{\prime}\right\}$ is a Cauchy sequence in $L^{2}$, which shows that $(H-i)^{-1} Q\left(H_{0}-i\right)^{-1}$ is completely continuous.

Q.E.D.

When we discuss the principle of limiting absorption for $H=H_{0}+Q$ in $\S 4$, we want to claim that $H$ has no eigenvalues on the intervals $(-\infty,-1)$ and $(+1,+\infty)$. So we shall give a sufficient condition in the following.

Proposition 2.5. Let $Q(x)$ have $C^{1}$ components $q_{i j}(x)$ except at a 
finite number of singularities, and let there exist positive numbers $R_{0}$ and $p>3$ such that

$$
\begin{gathered}
\int_{B\left(R_{0}\right)}|Q(x)|^{p} d x<+\infty, \\
\sum_{k=1}^{3}\left|\frac{\partial Q}{\partial x_{k}}\right| \leqq \text { const. } \quad\left(|x| \geqq R_{0}\right)
\end{gathered}
$$

and

$$
|x||Q(x)| \rightarrow 0 \quad(|x| \rightarrow \infty)
$$

Then $H=H_{0}+Q$ has no eigenvalues on $(-\infty,-1)$ and $(+1,+\infty)$.

For the proof of the above proposition we need only to follow Roze [9], where a similar result is proved.

\section{§3. An Estimate for the Resolvent of $\boldsymbol{H}_{0}$}

In this section we shall study the behavior of the resolvent of $H_{0}$ near the real axis.

The following proposition is a special case of Agmon [1], Theorem 2.2 .

Proposition 3.1. For any $s>\frac{1}{2}$ and $b>a>0$, there exists $a$ positive number $C_{2}=C_{2}(s, a, b)$ such that

$$
\|u\|_{H_{-s}^{2}} \leqq C_{2}\|(-\Delta-\lambda) u\|_{L_{s}^{2}}
$$

(see below for $H_{s}^{2}$ and $L_{s}^{2}$ ) for all $u \in H_{s}^{2}$ and $\lambda \in K(a, b)$, where $K(a, b)$ denotes the set of all complex numbers such that $a \leqq \operatorname{Re} \lambda \leqq b,|\operatorname{Im} \lambda| \leqq 1$ $(\operatorname{Re} \lambda(\operatorname{Im} \lambda)$ is the real (imaginary) part of $\lambda) . \quad C_{2}$ is independent of $u$ and $\lambda$.

For $s$ a real number, $L_{s}^{2}$ denotes the Hilbert space of all complexvalued functions $u(x)$ defined in $\mathbf{R}^{3}$ such that $(1+|x|)^{s} u(x) \in L^{2}\left(\mathbf{R}^{3}\right)$ with the norm 


$$
\|u\|_{L_{s}^{2}}=\left(\int_{\mathbf{R}^{3}}(1+|x|)^{2 s}|u(x)|^{2} d x\right)^{\frac{1}{2}}
$$

For $m$ a non-negative integer and $s$ a real number. $H_{s}^{m}$ denotes the class of all $L_{s}^{2}$ functions with distribution derivatives in $L_{s}^{2}$ up to the $m$-th order inclusive, which is normed by

$$
\|u\|_{H_{s}^{m}}=\left(\int_{\mathbf{R}^{3}}(1+|x|)^{2 s} \sum_{|\alpha| \leqq m}\left|D^{\alpha} u\right|^{2} d x\right)^{\frac{1}{2}}
$$

where $D^{\alpha} u(x)=\frac{\partial^{\alpha_{1}+\alpha_{2}+\alpha_{3}}}{\partial x_{1} \partial x_{2} \partial x_{3}} u\left(x_{1}, x_{2}, x_{3}\right)$ and $|\alpha|=\left|\left(\alpha_{1}, \alpha_{2}, \alpha_{3}\right)\right|=\alpha_{1}+$ $\alpha_{2}+\alpha_{3}$. For $t$ a real number, $H^{t}$ denotes the class of all temperate distributions whose Fourier transforms are $L_{t}^{2}$ functions. We define the norm in $H^{t}$ by

$$
\|u\|_{H^{t}}=\|\hat{u}(\xi)\|_{L_{t}^{2}}
$$

where $\hat{u}(\xi)$ is the Fourier transform of $u$ :

$$
\hat{u}(\xi)=(2 \pi)^{-\frac{3}{2}} \int_{\mathbf{R}^{3}} e^{-i<\xi, x>} u(x) d x .
$$

It is well known that $H^{t}$ coincides with the class of $L^{2}\left(\mathbf{R}^{3}\right)$ functions with distribution derivatives in $L^{2}\left(\mathbf{R}^{3}\right)$ up to the $t$-th order inclusive when $t$ is a non-negative integer.

The next proposition will be often used in the present paper.

Proposition 3.2. Let $\varphi(x)$ be a complex-valued function defined in $\mathbf{R}^{3}$ such that $\varphi(x)$ has bounded continuous partial derivatives up to the $m$-th order inclusive, where $m$ is a natural number. Take an arbitrary real number $s$ such that $0 \leqq s \leqq m$. Then we obtain

$$
\varphi(x) f(x) \in H^{s}
$$

for $f(x) \in H^{s}$. There exists a positive constant $C_{3}$ such that

$$
\|\varphi(x) f(x)\|_{H^{s}} \leqq C_{3}\|f(x)\|_{H^{s}}
$$

for $f \in H^{s}\left(\mathbf{R}^{3}\right)$. 
The proof of the above proposition follows almost trivially when $s$ is an integer. The general case when $s$ is not necessarily integral can be reduced to the integral case by using an interpolation theorem (see, e.g., Gagliardo [5]).

For $-\infty<a<b<-1$ or $+1<a<b<+\infty, J_{+}(a, b)\left(J_{-}(a, b)\right)$ denotes the set of all complex numbers $\lambda$ such that $a \leqq \operatorname{Re} \lambda \leqq b$ and $1>\operatorname{Im} \lambda>0$ $(0>\operatorname{Im} \lambda>-1)$. We put

$$
J(a, b)=J_{+}(a, b) \cup J_{-}(a, b) .
$$

Now the following theorem holds.

Theorem 3.1. For any $s>\frac{1}{2}$ and $J(a, b)$, there exists a positive constant $C_{6}=C_{6}(s, a, b)$ such that

$$
\|u\|_{H_{-s}^{1}} \leqq C_{6}\left\|\left(L_{0}-\lambda\right) u\right\|_{L_{s}^{2}}
$$

for all $u \in H_{s}^{1}$ and $\lambda \in J(a, b)$, where $C_{6}$ is independent of $u$ and $\lambda_{.} H_{s}^{1}$ $\left(L_{s}^{2}\right)$ here denotes the space of all $C^{4}$-valued functions with the components in $H_{s}^{1}\left(L_{s}^{2}\right)$.

Proof. It is enough to show that the inequality (3.2) holds for all $u \in C_{0}^{\infty}$, because $C_{0}^{\infty}$ is dense in $H_{s}^{1}$. We put

$$
f=\left(L_{0}-\lambda\right) u
$$

for $u \in C_{0}^{\infty}$ and $\lambda \in J(a, b)$. Then we obtain by taking the Fourier transforms of both sides,

$$
\left(\hat{L}_{0}(\xi)-\lambda\right) \hat{u}(\xi)=\hat{f}(\xi),
$$

where $\hat{L}_{0}(\xi)$ was defined in $\S 2$. From Proposition 2.2 we have

$$
\left(\hat{L}_{0}(\xi)-\lambda\right)^{-1}=\frac{\widehat{L}_{0}(\xi)+\lambda}{|\xi|^{2}+1-\lambda^{2}}
$$

So (3.4) shows that 


$$
u(x)=(2 \pi)^{-\frac{3}{2}} \int_{\mathbf{R}^{3}} e^{i<x, \xi\rangle} \frac{\hat{L}_{0}(\xi)+\lambda}{|\xi|^{2}+1-\lambda^{2}} \hat{f}(\xi) d \xi .
$$

Next we take $\gamma(x) \in C_{0}^{\infty}\left(\mathbf{R}^{3}\right)$ such that

$$
\gamma(x)=1 \text { for }|x|^{2}+1 \leqq \max \left(a^{2}, b^{2}\right)+\delta, \quad(\delta>0) .
$$

Then (3.5) is represented as

$$
\begin{aligned}
u(x)= & (2 \pi)^{-\frac{3}{2}} \int_{\mathbf{R}^{3}} e^{i<x, \xi>\frac{\gamma(\xi)\left(\widehat{L}_{0}(\xi)+\lambda\right)}{|\xi|^{2}+1-\lambda^{2}} \hat{f}(\xi) d \xi} \\
& +(2 \pi)^{-\frac{3}{2}} \int_{\mathbf{R}^{3}} e^{i<x, \xi>} \frac{(1-\gamma(\xi))\left(\widehat{L}_{0}(\xi)+\lambda\right)}{|\xi|^{2}+1-\lambda^{2}} \hat{f}(\xi) d \xi \\
\equiv & u_{1}(x)+u_{2}(x) .
\end{aligned}
$$

First we estimate $u_{1}(x)$. We denote by $\Lambda(x)$ the inverse Fourier transform of $\gamma(\xi)\left(\hat{L}_{0}(\xi)+\lambda\right) \hat{f}(\xi)$. Then

$$
\left(-\Delta+1-\lambda^{2}\right) u_{1}(x)=\Lambda(x) .
$$

$u_{1}(x)$ belongs to $H_{s}^{2}$, as $\frac{\xi^{\alpha} \gamma(\xi)\left(\hat{L}_{0}(\xi)+\lambda\right)}{|\xi|^{2}+1-\lambda^{2}} \hat{f}(\xi)$ belongs to $H^{s}$ for $|\alpha| \leqq 2$ by means of Proposition 3.2. Therefore according to Propositions 3.1 and 3.2 ,

$$
\left\|u_{1}\right\|_{H_{-s}^{2}} \leqq C_{7} \bar{\Pi} \Lambda(x)\left\|_{L_{s}^{2} \leqq C_{8}}\right\| f \|_{L_{s}^{2}}
$$

where positive constants $C_{7}, C_{8}$ are dependent on $s>\frac{1}{2}$ and $J$. To estimate $u_{2}$ we note that there is a positive constant $C_{9}=C_{9}(J)$ such that

$$
\left|\frac{\xi^{\alpha}(1-\gamma(\xi))\left(\widehat{L}_{0}(\xi)+\lambda\right)}{|\xi|^{2}+1-\lambda^{2}}\right| \leqq C_{9}
$$

for $\xi \in \mathbf{R}^{3},|\alpha| \leqq 1$ and $\lambda \in J$. Then we have

$$
\left\|u_{2}\right\|_{H_{-s}^{1}} \leqq\left\|u_{2}\right\|_{H^{1}} \leqq C_{10}\|f\|_{L^{2}} \leqq C_{10}\|f\|_{L_{s}^{2}}
$$

The desired estimate is obtained from (3.7) and (3.8).

Q.E.D. 


\section{§4. The Principle of Limiting Absorption for $H=H_{0}+Q$}

In this section we shall show that the assertion of Theorem 3.1 holds for $H=H_{0}+Q$ if $Q(x)$ satisfies the condition (A) to be stated later, and prove the principle of limiting absorption and the absolute continuity for $H$. We start with proving two lemmas.

Lemma 4.1. The following inequality is valid for all $\varphi \in L^{2}\left(B_{r+1}\right)$ such that $L_{0} \varphi \in L^{2}\left(B_{r+1}\right)$ :

$$
\|\varphi\|_{H^{1}\left(B_{r}\right)} \leqq C_{11}\left(\|\varphi\|_{L^{2}\left(B_{r+1}\right)}+\left\|L_{0} \varphi\right\|_{L^{2}\left(B_{r+1}\right)}\right)
$$

where $C_{11}$ is independent of $r>0$.

Proof. We can choose $\nu_{r} \in C_{0}^{\infty}\left(\mathbf{R}^{3}\right)$ as follows :

$$
\begin{aligned}
& \nu_{r}(x)=\left\{\begin{array}{l}
1,|x| \leqq r \\
0,|x| \geqq r+1,
\end{array}\right. \\
& \max _{\substack{j=1,2,2,3 \\
x \in \mathbb{R}^{3}}}\left|\frac{\partial}{\partial x_{j}} \nu_{r}(x)\right| \leqq C_{12},
\end{aligned}
$$

where the positive constant $C_{12}$ is independent of $r>0$, Then

$$
L_{0}\left(\nu_{r} \varphi\right)=\nu_{r} L_{0} \varphi+\frac{1}{i} \sum_{j=1}^{3} \alpha_{j}\left(\frac{\partial}{\partial x_{j}} \nu_{r}(x)\right) \varphi
$$

Thus we obtain $\left\|L_{0}\left(\nu_{r} \varphi\right)\right\|_{L^{2}}<+\infty$, which shows that $\nu_{r} \varphi$ belongs to $H^{1}$. Therefore we have $\|\varphi\|_{H^{1}\left(B_{r}\right)} \leqq\left\|\nu_{r} \varphi\right\|_{H^{1}} \leqq C_{13}\left\|L_{0}\left(\nu_{r} \varphi\right)\right\|_{L^{2}} \leqq C_{14}$ $\left(\left\|L_{0} \varphi\right\|_{L^{2}\left(B_{r+1}\right)}+\|\varphi\|_{L^{2}\left(B_{r+1}\right)}\right)$, when $C_{13}$ and $C_{14}$ are independent of $r>0$.

Q.E.D.

Throughout this section we assume that $Q(x)$ satisfies the following condition:

(A) $Q(x)$ is a $4 \times 4$ Hermitian matrix valued function with $C^{1}$ 
components $q_{i j}(x)$ except at a finite number of singularities and there exist positive numbers $h, R_{0}$ and $p>3$ such that

$$
\begin{gathered}
\int_{B\left(R_{0}\right)}|Q(x)|^{p} d x<+\infty, \\
\sum_{k=1}^{3}\left|\frac{\partial Q}{\partial x_{k}}\right| \leqq \text { const. } \quad\left(|x| \geqq R_{0}\right)
\end{gathered}
$$

and

$$
|Q(x)| \leqq \frac{\text { const. }}{(1+|x|)^{1+h}} \quad\left(|x| \geqq R_{0}\right)
$$

Lemma 4.2. Let (A) be satisfied and suppose $\frac{1}{2}<s<\frac{1+h}{2}$. Then there is a positive constant $C_{15}=C_{15}(s, a, b)$ such that

$$
\left(\|u\|_{H_{-s}^{1}}\right)^{2} \leqq C_{15}\left(\|(L-\lambda) u\|_{L_{s}^{2}}^{2}+\int_{B_{R}}|u(x)|^{2} d x\right)
$$

for all $u \in H_{s}^{1}$ and $\lambda \in J(a, b)$, where $R$ is a positive number such that $R>R_{0}$.

Proof. From Theorem 3.1 we have

$$
\begin{aligned}
& \left(\|u\|_{H_{-s}^{1}}\right)^{2} \leqq C_{6}^{2}\left\|\left(L_{0}-\lambda\right) u\right\|_{L_{s}^{2}}^{2} \\
& \leqq 2 C_{6}^{2}\left(\|(L-\lambda) u\|_{L_{s}^{2}}^{2}+\|Q u\|_{L_{s}^{2}}^{2}\right) .
\end{aligned}
$$

From (2.4) of Corollary 2.1 and (4.2), we have

$$
\begin{aligned}
\|Q u\|_{L_{s}^{2}}^{2} \leqq \varepsilon\|u\|_{H^{1}\left(B_{R_{0}}+1\right)}^{2}+C_{16}(\varepsilon, R) \int_{B_{R}}|u(x)|^{2} d x \\
+C_{17}(1+R)^{-2 h+4 s-2} \int_{E_{R}}(1+|x|)^{-2 s}|u(x)|^{2} d x
\end{aligned}
$$

where $R>R_{0}$ and $\varepsilon>0$. Thus we can obtain (4.3) from (4.4) and (4.5) if we take sufficiently small $\varepsilon>0$ and sufficiently large $R$ by virtue of 


$$
-2 h+4 s-2=4\left(s-\frac{1+h}{2}\right)<0
$$

Q.E.D.

Theorem 4.1. Let $Q(x)$ satisfy the condition $(A)$. Then for any $s>\frac{1}{2}$ and $J_{+}(a, b)$, there exists a positive constant $C_{18}=C_{18}(s, a, b)$ such that

$$
\|u\|_{H_{-s}^{1}} \leqq C_{18}\|(L-\lambda) u\|_{L_{s}^{2}}
$$

for all $u \in H_{s}^{1}$ and $\lambda \in J_{+}(a, b)$, where $L=L_{0}+Q(x) . \quad C_{15}$ is independent of $u$ and $\lambda$. A similar result holds with $J_{+}(a, b)$ replaced by $J_{-}(a, b)$.

Proof. It suffices to show that (4.6) holds for $\frac{1}{2}<s<\frac{1+h}{2}$, which we always assume hereafter. Furthermore, in view of Lemma 4.2 we have only to show that there is a positive constant $C_{19}=C_{19}(s, a, b)$ such that

$$
\int_{|x| \leqq R}|u(x)|^{2} d x \leqq C_{19}\|(L-\lambda) u\|_{L_{s}^{2}}^{2}
$$

for all $u \in H_{s}^{1}$ and $\lambda \in J_{+}(a, b)$.

It we assume the contrary, there should be a sequence $\left\{u_{n}\right\}$ of $H_{s}^{1}$ and a sequence $\left\{\lambda_{n}\right\}$ of $J_{+}(a, b)$ such that

$$
\begin{gathered}
\int_{|x| \leqq R}\left|u_{n}(x)\right|^{2} d x=1 \quad(n=1,2, \cdots), \\
\left(L-\lambda_{n}\right) u_{n} \rightarrow 0 \text { in } L_{s}^{2} \quad(n \rightarrow \infty) .
\end{gathered}
$$

We may assume that $\lambda_{n} \rightarrow \lambda_{0}$, where $\lambda_{0}$ is a real number with $\lambda_{0} \in[a, b]$ because if $\operatorname{Im} \lambda_{0}>\eta_{0}>0$, we would obtain for $n$ large

$$
\int_{|x| \leqq R}\left|u_{n}(x)\right|^{2} d x \leqq\left\|u_{n}\right\|_{L^{2}}^{2} \leqq \eta_{0}^{-2}\left\|\left(L-\lambda_{n}\right) u_{n}\right\|_{L^{2}}^{2}
$$

which is inconsistent with (4.8) and (4.9).

From (4.3) of Lemma 4.2 and (4.8) we have 


$$
\left\|u_{n}\right\|_{H_{-s}^{1}} \leqq C_{20} \quad(n=1,2, \cdots)
$$

where $C_{20}$ is a positive constant. If follows from (4.10) and Rellich's theorem that we can select a subsequence from $\left\{u_{n}\right\}$ which converges locally in $L^{2}$. We shall denote this subsequence by $\left\{u_{n}\right\}$ again. We put

$$
f_{n}=\left(L-\lambda_{n}\right) u_{n}
$$

Then evidently we have from (4.9)

$$
f_{n} \rightarrow 0 \text { in } L_{s}^{2}
$$

and

$$
L_{0} u_{n}=-Q u_{n}+\lambda_{n} u_{n}+f_{n}
$$

Then it follows from (4.5), which appeared in the proof of Lemma 4.2, (4.10) and $\frac{1}{2}<s<\frac{1+h}{2}$ that

$$
Q u_{n} \rightarrow Q u_{0} \text { in } L_{s}^{2} \quad(n \rightarrow \infty) .
$$

Therefore from Lemma 4.1, (4.11), (4.12), (4.13) and the fact that $\left\{u_{n}\right\}$ converges locally in $L^{2}$, we have

$$
u_{n} \rightarrow u_{0} \text { locally in } H^{1} \text {. }
$$

Thus we have from (4.8)

$$
\int_{|x| \leqq R}\left|u_{0}(x)\right|^{2} d x=1
$$

and

$$
\left(L_{0}+Q(x)\right) u_{0}(x)=\lambda_{0} u_{0}(x)
$$

If we can show $u_{0} \in L^{2}$, then $u_{0}=0$ follows from Proposition 2.5. This contradicts (4.15), which concludes the proof of our theorem.

We shall prove $u_{0} \in L^{2}$. We put

$$
g_{n}=f_{n}-Q u_{n}, \quad g_{0}=-Q u_{0} .
$$


From (4.11) and (4.13), we have

$$
g_{n} \rightarrow g_{0} \text { in } L_{s}^{2}
$$

We may assume $u_{n} \rightarrow u_{0}$ in $H_{-s}^{1}$ from (4.10), where $\rightarrow$ denotes the weak convergence. Then we have

$$
\left(u_{n}, g_{n}\right) \rightarrow\left(u_{0}, g_{0}\right)=-\left(u_{0}, Q u_{0}\right) \quad \text { as } n \rightarrow \infty \text {, }
$$

where $(\cdot, \cdot)$ denotes the usual inner product $(f, g)=\int_{\mathbf{R}^{3}}\langle f(x), g(x)\rangle d x$.

On the other hand, $\left(u_{n}, g_{n}\right)$ is represented in terms of Fourier transforms as follows:

$$
\begin{aligned}
\left(u_{n}, g_{n}\right) & =\left(\hat{u}_{n}, \hat{g}_{n}\right)=\int_{\mathbf{R}^{3}} \frac{\left.<\left(\hat{L}_{0}(\xi)+\lambda_{n}\right) \hat{g}_{n}, \hat{g}_{n}\right\rangle}{|\xi|^{2}+1-\lambda_{n}^{2}} d \xi \\
& =\int_{0}^{+\infty} \frac{1}{r^{2}+1-\lambda_{n}^{2}} d r \int_{S_{r}}<\left(\hat{L}_{0}(\xi)+\lambda_{n}\right) \hat{g}_{n}, \hat{g}_{n}>d S
\end{aligned}
$$

where $S_{r}$ denotes the spherical surface of radius $r$ about the origin. It can be shown by using Sobolev's imbedding theorem for $H^{s}\left(\mathbf{R}^{3}\right), s>\frac{1}{2}$, that $\int_{S_{r}}<\left(\hat{L}_{0}(\xi)+\lambda_{n}\right) \hat{g}_{n}(\xi), \hat{g}_{n}(\xi)>d S$ is locally Hölder continuous uniformly with respect to $r$ (see, e.g., Sobolev [10]). This enables us to take the limit for $n \rightarrow \infty$ in (4.18) and to obtain

$$
\begin{aligned}
& \lim _{n \rightarrow \infty}\left(u_{n}, g_{n}\right)=\frac{\pi i}{2 \sqrt{\lambda_{0}^{2}-1}} \int_{S \sqrt{\lambda_{0}^{2}-1}}<\left(\hat{L}_{0}(\xi)+\lambda_{0}\right) \hat{g}_{0}, \hat{g}_{0}>d S \\
& +\lim _{\eta \downarrow 0}\left[\int_{|\xi| \leqq \sqrt{\lambda_{0}^{2}-1-\eta}} \frac{<\left(\widehat{L}_{0}(\xi)+\lambda_{0}\right) \hat{g}_{0}, \hat{g}_{0}>}{|\xi|^{2}+1-\lambda_{0}^{2}} d \xi+\int_{|\xi| \leqq \sqrt{\lambda_{0}^{2}-1+\eta}} \underset{\ldots \ldots}{\mid .}\right.
\end{aligned}
$$

As $\left(u_{n}, g_{n}\right)$ converges by $(4.17)$ to $-\left(u_{0}, Q u_{0}\right)$, which is a real number, we have from the above relation

$$
\int_{S_{\sqrt{\lambda_{0}^{2}-1}}}<\left(\hat{L}_{0}(\xi)+\lambda_{0}\right) \hat{g}_{0}, \hat{g}_{0}>d S=0
$$

This implies

$$
\left(\hat{L}_{0}(\xi)+\lambda_{0}\right) \hat{g}_{0}(\xi)=0 \quad \text { on }|\xi|=\sqrt{\lambda_{0}^{2}-1}
$$


Indeed, let $\left\{\psi_{j}(\xi)\right\}_{j=1,2,3,4}$ be an orthonormal system such that $\psi_{1}(\xi)$, $\psi_{2}(\xi)$ are eigenvectors associated with $+\sqrt{|\xi|^{2}+1}$ and $\psi_{3}(\xi), \psi_{4}(\xi)$ with $-\sqrt{|\xi|^{2}+1}$. Then

$$
\hat{L}_{0}(\xi) \hat{g}_{0}(\xi)=\sqrt{|\xi|^{2}+1} \sum_{j=1}^{2}<\hat{g}_{0}, \psi_{j}>\psi_{j}-\sqrt{|\xi|^{2}+1} \sum_{j=3}^{4}<\hat{g}_{0}, \psi_{j}>\psi_{j} .
$$

$\lambda_{0}$ is $+\sqrt{|\xi|^{2}+1}$ or $-\sqrt{|\xi|^{2}+1}$ when $|\xi|=\sqrt{\lambda_{0}^{2}-1}$. Therefore, we have

$$
<\left(\hat{L}_{0}(\xi)+\lambda_{0}\right) \hat{g}_{0}, \hat{g}_{0}>= \begin{cases}+2 \sqrt{|\xi|^{2}+1} \sum_{j=1}^{2}\left|<\hat{g}_{0}, \psi_{j}>\right|^{2} \quad\left(\lambda_{0}=+\sqrt{|\xi|^{2}+1}\right), \\ -2 \sqrt{|\xi|^{2}+1} \sum_{j=3}^{4}\left|<\hat{g}_{0}, \psi_{j}>\right|^{2} \quad\left(\lambda_{0}=-\sqrt{|\xi|^{2}+1}\right) .\end{cases}
$$

From (4.19), we have thus

$$
<\hat{g}_{0}, \psi_{j}>=0 \text { on }|\xi|=\sqrt{\lambda_{0}^{2}-1}
$$

where $j=1,2$ in case of $\lambda_{0}=+\sqrt{|\xi|^{2}+1}$ and $j=3,4$ in case of $\lambda_{0}=$ $-\sqrt{|\xi|^{2}+1}$, which yields (4.20).

Next we shall show

$$
\hat{u}_{0}(\xi)=\frac{\left(\hat{L}_{0}(\xi)+\lambda_{0}\right) \hat{g}_{0}(\xi)}{|\xi|^{2}+1-\lambda_{0}^{2}} \quad \text { for almost every } \xi \in \mathbb{R}^{3}
$$

To prove this we shall take arbitrary $\varphi(\xi) \in C_{0}^{\infty}$. Then we have

$$
\left(\hat{u}_{n}(\xi), \varphi(\xi)\right) \rightarrow\left(\hat{u}_{0}(\xi), \varphi(\xi)\right)
$$

because $u_{n} \rightarrow u_{0}$ in $H_{-s}^{1}$, which implies $\hat{u}_{n} \rightarrow \hat{u}_{0}$ in $H^{-s}$. On the other hand, we have for $n \rightarrow \infty$

$$
\begin{aligned}
\left(\hat{u}_{n}(\xi), \varphi(\xi)\right)=\int_{\mathbf{R}^{3}} & \frac{\left\langle\left(\hat{L}_{0}(\xi)+\lambda_{n}\right) \hat{g}_{n}, \varphi>\right.}{|\xi|^{2}+1-\lambda_{n}^{2}} d \xi \\
& \rightarrow \text { p.v. } \int_{\mathbf{R}^{3}} \frac{\left\langle\left(\hat{L}_{0}(\xi)+\lambda_{0}\right) \hat{g}_{0}, \varphi>\right.}{|\xi|^{2}+1-\lambda_{0}^{2}} d \xi
\end{aligned}
$$

where p.v. denotes the principal value. This is obtained from (4.20) and the local Hölder continuity of $\int_{S_{r}}<\left(\hat{L}_{0}(\xi)+\lambda_{0}\right) \hat{g}_{0}(\xi), \varphi>d S$ with respect to $r \in(0,+\infty)$, which can be shown by using again Sobolev's imbedding theorem and the fact $g_{0} \in L_{s}^{2}$, i.e., $\hat{g}_{0} \in H^{s}$. (4.21) now follows in view of the arbitrariness of $\varphi$. 
By (4.20) and (4.21) we can show that

$$
\hat{u}_{0}(\xi)=\frac{\left(\hat{L}_{0}(\xi)+\lambda_{0}\right) \hat{g}_{0}(\xi)}{|\xi|^{2}+1-\lambda_{0}^{2}} \in H^{s-1},
$$

if we make use of the following proposition, which was referred to by S. Agmon in his lectures at the Oberwolfach Symposium on Mathematical Theory of Scattering, 1971.

Proposition 4.1. Let $g \in H^{t}\left(\mathbf{R}^{n}\right)$ satisfy

$$
g(\xi)=0 \text { on }|\xi|=\lambda \text {, }
$$

where $t>\frac{1}{2}$ and $\lambda>0$. Then we have

$$
\frac{g(\xi)}{|\xi|-\lambda} \in H^{t-1}\left(\mathbf{R}^{n}\right)
$$

Let us prove (4.22). We shall take $\gamma(\xi) \in C_{0}^{\infty}\left(\mathbf{R}^{3}\right)$ such that

$$
\gamma(\xi)=1 \quad \text { for } \frac{1}{2} \sqrt{\lambda_{0}^{2}-1} \leqq|\xi| \leqq \frac{3}{2} \sqrt{\lambda_{0}^{2}-1}
$$

Then $\hat{u}_{0}(\xi),(4.21)$, is written as

$$
\begin{aligned}
\hat{u}_{0}(\xi)= & \frac{1}{|\xi|-\sqrt{\lambda_{0}^{2}-1}} \cdot \frac{\gamma(\xi)\left(\hat{L}_{0}(\xi)+\lambda_{0}\right) \hat{g}_{0}(\xi)}{|\xi|+\sqrt{\lambda_{0}^{2}-1}} \\
& +(1-\gamma(\xi)) \frac{\left(\hat{L}_{0}(\xi)+\lambda_{0}\right) \hat{g}_{0}(\xi)}{|\xi|^{2}+1-\lambda_{0}^{2}}
\end{aligned}
$$

We obtain by Proposition 3.2

$$
\frac{\gamma(\xi)\left(\hat{L}_{0}(\xi)+\lambda_{0}\right)}{|\xi|+\sqrt{\lambda_{0}^{2}-1}} \hat{g}_{0}(\xi) \in H^{s},
$$

because $g_{0}(x) \in L_{s}^{2}$ and, therefore, $\hat{g}_{0}(\xi) \in H^{s}$. Thus we can apply in view of (4.20) Proposition 4.1 to the first term of (4.24). For the second term we have from Proposition 3.2 and the property of $\gamma(\xi)$

$$
(1-\gamma(\xi)) \frac{\left(\hat{L}_{0}(\xi)+\lambda_{0}\right)}{|\xi|^{2}+1-\lambda_{0}^{2}} \hat{g}_{0}(\xi) \in H^{s} .
$$


Thus we obtain (4.22).

$\hat{u}_{0}(\xi) \in H^{s-1}$ implies $u_{0}(x) \in L_{s-1}^{2}$, and hence $g_{0}=-Q u_{0} \in \frac{L_{\frac{4 s-1}{2}}^{2}}{\text { by }}$ (4.2) and $\frac{1}{2}<s<\frac{1+h}{2}$. Another use of Proposition 4.1 gives $\hat{u}_{0}(\xi) \in$ $H^{\frac{4 s-3}{2}}$ by means of $(4.20)$ and $\hat{g}_{0}(\xi) \in H^{\frac{4 s-1}{2}}$. If we repeat this procedure, we obtain finally $u_{0}(x) \in L^{2}$. Then we have $u_{0}=0$ from Proposition 2.5 and (4.16), which contradicts (4.15). The proof of Theorem 4.1 is now complete.

Q.E.D.

Corollary 4.1. (The principle of limiting absorption). Let $Q(x)$ satisfy the condition (A). We put

$$
u_{z}(f)=(H-z)^{-1} f
$$

for $f \in L_{s}^{2}$ and $\operatorname{Im} z \neq 0$, where $s>\frac{1}{2}$. Then for any real number $\lambda$ such that $|\lambda|>1$, there exists $u^{+}(\lambda, f)$ such that

$$
u_{z}(f) \rightarrow u^{+}(\lambda, f) \quad \text { in } H_{-s}^{1}
$$

as $z \rightarrow \lambda+0 i$. Similarly there exists $u^{-}(\lambda, f)$ such that

$$
u_{z}(f) \rightarrow u^{-}(\lambda, f) \text { in } H_{-s}^{1}
$$

as $z \rightarrow \lambda-0 i . \quad u^{+}(\lambda, f)$ and $u^{-}(\lambda, f)$ are solutions of the equation

$$
\left(L_{0}+Q(x)\right) u(x)=\lambda u(x)+f(x)
$$

and they are continuous functions of $\lambda$ in the topology of $H_{-s}^{1}$.

Proof. As before we may assume $\frac{1+h}{2}>s>\frac{1}{2}$. We take an arbitary sequence $\left\{z_{n}\right\}$ such that $\operatorname{Im} z_{n}>0$ and $z_{n} \rightarrow \lambda+0 i$ as $n \rightarrow \infty$. Then we have by Proposition 3.2,

$$
\xi^{\alpha} u_{z_{n}}(f)^{\wedge}(\xi)=\frac{\xi^{\alpha}\left(\hat{L}_{0}(\xi)+\lambda\right)}{|\xi|^{2}-z_{n}^{2}+1} \hat{g}_{z_{n}}(\xi) \in H^{s}
$$

for $|\alpha| \leqq 1$, where $g_{z_{n}}=-Q u_{z_{n}}+f \in L_{s}^{2}$. This implies $u_{z_{n}}(f) \in H_{s}^{1}$. We have, therefore, from Theorem 4.1 


$$
\left\|u_{z_{n}}(f)\right\|_{H_{-s}^{\mathbf{1}}} \leqq C_{21}\|f\|_{L_{s}^{2}}
$$

for $n$ large and $s>\frac{1}{2}$, where $C_{21}$ is a positive constant independent of $z_{n}$. We can select a subsequence $\left\{u_{z_{n}^{\prime}}\right\}$ from the sequence $\left\{u_{z_{n}}\right\}$ which converges in the sense of $H_{-s}^{1}$ to some function $u_{0}$ by the method described in the proof of Theorem 4.1.

Next we want to obtain

$$
u_{z_{n}} \rightarrow u_{0} \text { in } H_{-s}^{1}
$$

If we assume the contrary, there would exist a positive constant $\delta_{0}$ and a subsequence $\left\{z_{n}^{\prime \prime}\right\}$ of the sequence $\left\{z_{n}\right\}$ such that

$$
\left\|u_{z_{n}^{\prime \prime}}-u_{0}\right\|_{H_{-s}^{1}}>\delta_{0}>0
$$

We can choose a subsequence $\left\{u_{z_{n}^{\prime \prime}}\right\}$ of the sequence $\left\{u_{z_{n}^{\prime \prime}}\right\}$ which tends in $H_{-s}^{1}$ to some function $u_{1}$ by the above-mentioned argument. We put

$$
v_{n}=u_{z_{n}^{\prime}}-u_{z_{n}^{\prime \prime}}
$$

Then we have

$$
v_{n} \rightarrow u_{0}-u_{1} \text { in } H_{-s}^{1}
$$

and

$$
\left(L_{0}+Q(x)\right) v_{0}(x)=\lambda v_{0}(x),
$$

where $v_{0}=u_{0}-u_{1}$. Then $v_{0} \in L^{2}$ can be shown as in the proof of Theorem 4.1. Therefore, by Proposition 2.5 we obtain $v_{0}=0$, which contradicts (4.27). This proves (4.26).

We now show that the limit $u_{0}$ is independent of the choice of the sequence $\left\{z_{n}\right\}$ converging to $\lambda+0 i$. We take another sequence $\left\{\nu_{n}\right\}$ such that $\nu_{n} \rightarrow \lambda+0 i$, as $n \rightarrow \infty$. Then according to what we have established above, there exists $u_{2} \in H_{-s}^{1}$ such that $u_{\nu_{n}} \rightarrow u_{2}$ in $H_{-s}^{1}$. We shall show $u_{0}=u_{2}$. To prove this we put

$$
w_{n}=u_{z_{n}}-u_{\nu_{n}}, w_{0}=u_{0}-u_{2}
$$


Then we have

$$
w_{n} \rightarrow w_{0}=u_{0}-u_{2} \text { in } H_{-s}^{1}
$$

and

$$
\left(L_{0}+Q(x)\right) w_{0}=\lambda w_{0},
$$

which implies $w_{0}=0$ by using the same argument as the proof of (4.26). Therefore we have $u_{0}=u_{2}$.

Thus $u^{+}(\lambda, f)=u_{0}$ is well-defined for $f \in L_{s}^{2}$.

Finally we shall prove that $u^{+}(\lambda, f)$ is continuous in $\lambda$ with values in $H_{-s^{*}}^{1}$ In view of the fact that $u_{z}(f)$ is a continuous function of $z \in$ $J_{+}(a, b)$ in $H_{-s}^{1}$, as can be seen by using the resolvent equation, it suffices to prove that $u_{\lambda+i \eta}(f)$ tends in $H_{-s}^{1}$ to $u^{+}(\lambda, f)$, as $\eta \downarrow 0$, uniformly with respect to $\lambda \in[a, b]$. If we assume the contrary, there would be a positive number $\delta_{1}$ and two sequences $\left\{\lambda_{n}\right\}$ (contained in $[a, b]$ ) and $\left\{\eta_{n}\right\}$ such that $\eta_{n} \downarrow 0$ and

$$
\left\|u_{\lambda_{n}+i \eta_{n}}(f)-u^{+}\left(\lambda_{n}, f\right)\right\|_{H_{-s}^{1}} \geqq \delta_{1}>0
$$

On the other hand, we can select for each $n$ a real number $\eta_{n}^{\prime}$ such that $\eta_{n}^{\prime} \downarrow 0$ as $n \rightarrow \infty$, and

$$
\left\|u_{\lambda_{n}+i \eta_{n}^{\prime}}(f)-u^{+}\left(\lambda_{n}, f\right)\right\|_{H^{-1}-s}<\frac{\delta_{1}}{2}
$$

because $u_{\lambda+i \eta}(f) \rightarrow u^{+}(\lambda, f)$ in $H_{-s}^{1}$, as $\eta \downarrow 0$, for each $\lambda$. From (4.28) and (4.29) we have

$$
\left\|u_{\lambda_{n}+i \eta_{n}}(f)-u_{\lambda_{n}+i \eta_{n}^{\prime}}(f)\right\|_{H_{-s}^{1}} \geqq \frac{\delta_{1}}{2}>0
$$

As $[a, b]$ is a compact set, there is a convergent subsequence $\left\{\lambda_{n(j)}\right\}$ of $\left\{\lambda_{n}\right\}$. Denote by $\lambda_{0}$ the limit of $\left\{\lambda_{n(j)}\right\}$. Now (4.30) is inconsistent with the fact that $u_{\lambda_{n(j)}+i \eta_{n(j)}}(f)$ and $u_{\lambda_{n(j)}+i \eta_{j(n)}^{\prime}}(f)$ converge as $j \rightarrow \infty$ to the same limit $u^{+}\left(\lambda_{0}, f\right)$. Thus the convergence of $u_{\lambda+i \eta}(f)$ to $u^{+}(\lambda, f)$ for $\eta \downarrow 0$ is uniform.

Q.E.D. 
Corollary 4.2. Let $Q(x)$ satisfy the condition (A) and $E(\lambda)$ be the right-continuous resolution of the identity associated with $H=H_{0}+Q$. Then $(E(\lambda) f, f)$ is absolutely continuous on $(-\infty,-1)$ and $(+1,+\infty)$.

Proof. It is enough to show the assertion for all $f \in L_{s}^{2}\left(s>\frac{1}{2}\right)$, because $L_{s}^{2}$ is dense in $L^{2}$. We make use of the following relation,

$$
\begin{aligned}
& \frac{1}{2}((E(\beta)+E(\beta-0)) f, f)-\frac{1}{2}((E(\alpha)+E(\alpha-0)) f, f) \\
& \quad=\frac{1}{2 \pi i} \lim _{\eta \downarrow 0} \int_{\alpha}^{\beta}\left((H-\mu-i \eta)^{-1} f-(H-\mu+i \eta)^{-1} f, f\right) d \mu .
\end{aligned}
$$

We take an arbitary interval $[a, b]$ contained in $(-\infty,-1)$ or $(+1$, $+\infty)$. Then we have from Corollary 4.1 and (4.31)

$$
|(E(\beta) f, f)-(E(\alpha) f, f)|=\frac{1}{2 \pi i} \int_{\alpha}^{\beta}\left(u^{+}(\mu, f)-u^{-}(\mu, f), f\right) d \mu
$$

and

$$
|((E(\beta)-E(\alpha)) f, f)| \leqq C_{22}\|f\|_{L_{s}^{2}(\beta-\alpha)}^{2}
$$

for $a \leqq \alpha<\beta \leqq b$, where $C_{22}$ is a positive constant depending only on [a, $b]$ and $s$, which shows the desired absolute continuity.

Q.E.D.

In conclusion, the author wishes to express his sincere gratitude to Professor T. Ikebe for his enduring encouragements and valuable advices.

\section{References}

[1] Agmon, S., Spectral properties of Schrödinger operators, Actes Congrès intern. Math., 2 (1970), 679-683.

[2] Birman, M. S., On the spectrum of singular boundary problems, Math. Sb., 55 (1961), 125-174.

[3] Eidus, D. M., The principle of limiting absorption, Math. Sb., 57 (1962), 13-44: Trans. Amer. Math. Soc., 47 (1965), 157-191.

[4] Eidus, D. M., The principle of limit amplitude, Uspehi Math. Nauk, 24 (1969), 91-156: Russian Math. Surveys, 24 (1969), 97-167.

[5] Gagliardo, E., Interpolation d'espaces de Banach et applications, C. R. Acad. Sci. Paris, 248 (1959), 3517-3518. 
[6] Kato, T., Perturbation Theory for Linear Operators, Springer-Verlag, Berlin, 1966.

[7] Mochizuki, K., On the perturbation of the continuous spectrum of the Dirac operator, Proc. Japan Acad., 40 (1964), 707-712.

[8] Prosser, T., Relativistic potential scattering, J. Mathematical Phys. 4 (1963), 10481054.

[9] Rose, S.N., On the character of the spectrum of the Dirac operator, Theor. Math. Phys. 2 (1970), 377-382.

[10] Soboloev, S.L., Applications of Functional Analysis in Mathematical Physics, Amer. Math. Soc., Providence, 1963. 
